\title{
On Conway's Thrackle Conjecture
}

\author{
László Lovász* \\ Dept. Computer Science, Yale University \\ János Pach ${ }^{\dagger}$ \\ City College, CUNY and Courant Institute, NYU \\ Mario Szegedy \\ AT\&T Bell Laboratories
}

\begin{abstract}
A thrackle is a graph drawn in the plane so that its edges are represented by Jordan arcs and any two distinct arcs either meet at exactly one common vertex or cross at exactly one point interior to both arcs. About forty years ago, J. H. Conway conjectured that the number of edges of a thrackle cannot exceed the number of its vertices. We show that a thrackle has at most twice as many edges as vertices. Some related problems and generalizations are also considered.
\end{abstract}

\section{Introduction}

Let $G$ be a graph with vertex set $V(G)$ and edge set $E(G)$, and assume that it has no loops or multiple edges. A drawing of $G$ is a representation of $G$ in the plane such that every vertex corresponds to a point, and every edge is represented by a Jordan arc connecting the corresponding two points without passing through any other vertex. Two edges (arcs) are said to cross each other if they have an interior point $p$ in common. For simplicity, we always assume that no three edges cross at the same point. A crossing $p$ is called proper if in a small neighborhood of $p$ one edge passes from one side of the other edge to the other side. Due to its aesthetic appeal and wide range of

\footnotetext{
* Supported by NSF grant CCR-94-02916.

${ }^{\dagger}$ Supported by NSF grant CCR-91-22103, PSC-CUNY Research Award 663472 and OTKA-4269.
} 
applications in VLSI layout, computer-aided-design, software visualization, etc., the area of graph drawings has received a lot of attention in the past two decades. For a recent bibliography of graph drawing algorithms, see [DETT94].

There are many interesting results in topological graph theory characterizing all graphs embeddable on a given surface without crossing (see [WB78]). However, we know very little about the possible intersection patterns determined by the edges of a graph drawn on a surface. In particular, even for some very simple graphs we do not how to find the crossing number of $G$, i.e., the minimum number of crossing pairs of edges in a planar drawing of $G$. In the case when $G$ is a complete bipartite graph, this is Turán's brick factory problem [T77, G72]. The determination of the crossing number is known to be NP-complete [GJ83].

Another well-known open problem that illustrates our ignorance about graph drawings was raised by Conway about forty years ago. He defined a thrackle as a drawing of a graph $G$ with the property that any two distinct edges either

(i) share an endpoint, and then they do not have any other point in common; or

(ii) do not share an endpoint, in which case they meet exactly once and determine a proper crossing.

Thrackle Conjecture: The number of edges of a thrackle cannot exceed the number of its vertices.

A graph that can be drawn as a thrackle is said to be thrackleable. Assuming that the above conjecture is true, Woodall [W69] characterized all thrackleable graphs. With this assumption, a finite graph is thrackleable if and only if it has at most one odd cycle, it has no cycle of length four, and each of its connected components contains at most one cycle. Note that it is quite straightforward to check the necessity of these conditions (see Lemma 2.1). Using a construction suggested by Conway, the thrackle conjecture can be reduced to the following statement: If a graph $G$ consists of two even cycles meeting in a single vertex then $G$ is not thrackleable (cf. [W69, PRS94]). It is worth mentioning that the thrackle conjecture is true for straight-line thrackles, i.e., for drawings where every edge is represented by a segment [HP34, FS35, PA95]. See [LST94] for a surprising relation between straight-line thrackles and triangulations of certain polytopes, and [G75] for another geometric application.

Any two edges of a thrackle intersect in exactly one point, including the 
endpoints. For finite set-systems satisfying a similar condition we have the following well-known result [F40, BE48].

Fisher Inequality: Let $F$ be a family of subsets of a finite set $X$ such that any two members of $F$ have exactly one element in common. Then $F$ has at most as many members as the number of elements of $X$.

An interesting modular version of this inequality was discovered by Berlekamp [B69]. Suppose that every member of $F$ has an odd number of elements and that the intersection of any two members is even. Then $|F| \leq|X|$. These results and their generalizations originate in linear algebra and play a central role in finite geometries and in the theory of combinatorial designs (see [BF88]).

Since thrackles do not contain cycles of length four, it follows from [KST54] that the maximum number of edges a thrackle of $n$ vertices can have is $O\left(n^{3 / 2}\right)$. Our next theorem represents a substantial improvement on this bound.

Theorem 1.1 Every thrackle of $n$ vertices has at most $2 n-3$ edges.

The proof is based on the following result.

Theorem 1.2 Every thrackleable bipartite graph is planar.

Just like the Fisher inequality, the thrackle conjecture has some modular versions, too. For example, call a graph drawing a generalized (or modulo 2-) thrackle if any two edges meet an odd number of times, where "meet" means either "meet at a common vertex" or "meet at a proper crossing".

Theorem 1.3 Every generalized thrackle of $n$ vertices has at most $3 n-4$ edges.

Theorem 1.4 A bipartite graph can be drawn as a generalized thrackle if and only if it is planar.

Woodall [W72] asked whether the thrackle conjecture remains true for generalized thrackles. Our last theorem implies that the answer to this question is in the negative, because a bipartite planar graph of $n$ vertices can have as many as $2 n-4$ edges. 


\section{Three lemmas}

In the sequel, a thrackle and its underlying "abstract" graph are both denoted by $G$. If there is no danger of confusion, we make no notational distinction between a vertex (edge) of the graph and the corresponding point (arc).

Lemma 2.1 Let $G$ be a thrackleable graph. Then $G$ contains (i) no cycle of length four; (ii) no two vertex-disjoint odd cycles.

Proof: To show (ii), notice that a pair of vertex-disjoint odd cycles would be represented in a thrackle by two closed curves that properly cross each other an odd number of times.

Lemma 2.2 Let $C_{1}$ and $C_{2}$ be two cycles in a graph $G$ that have precisely one vertex $v$ in common. Suppose that $G$ can be drawn as a thrackle.

Then the two closed curves representing $C_{1}$ and $C_{2}$ cross each other in a small neighborhood of $v$ if and only if both cycles are odd.

Proof: Let $k_{i}$ denote the length of $C_{i}, i=1,2$. The closed curve representing $C_{1}$ divides the plane into $k_{1}\left(k_{1}-3\right) / 2+2$ connected cells. Color these cells with black and white so that no two cells that share a boundary arc have the same color. The curve representing $C_{2}$ intersects $C_{1}$ exactly $2\left(k_{1}-2\right)+\left(k_{2}-2\right) k_{1} \equiv k_{1} k_{2}$ times $(\bmod 2)$, not counting $v$. Every time $C_{2}$ intersects $C_{1}$, it passes from one cell to another whose color is different. Assume that in a small neighborhood of $v$ the initial segment of an edge of $C_{2}$ incident to $v$ lies in a white region. Then the initial segment of the other edge of $C_{2}$ incident to $v$ lies in a black region if and only if $k_{1} k_{2}$ is odd.

A graph consisting of three internally disjoint paths $P_{i}, i=1,2,3$ between $u$ and $v$ is called a $\Theta$-graph. A drawing of this $\Theta$-graph is said to be a preserver if in a small neighborhood of $u$ the initial pieces of the paths $P_{i}$ follow each other in the same circular order (clockwise or counterclockwise) as the final pieces do around $v$. Otherwise, the drawing is called a converter. Note that, using this terminology, if $G$ is a planar graph drawn in the plane without crossing then any $\Theta$-subgraph of this drawing is a converter.

The proof of the next lemma is very similar to that of the previous one.

Lemma 2.3 A $\Theta$-subgraph of a thrackle is a converter if and only if at most one of its three paths has odd length.

Remark: With the exception of Lemma 2.1(i), all statements and proofs in this section remain valid for generalized thrackles. 


\section{$3 \quad$ Bipartite thrackles}

Proof of Theorem 1.2: By Kuratowski's theorem, it is sufficient to show that a thrackleable bipartite graph $G$ does not contain a subdivision of $K_{5}$ or of $K_{3,3}$.

Suppose that $G$ contains a subdivision of $K_{5}$, whose vertices are $v_{0}, \ldots, v_{4}$. Assume without loss of generality that in a thrackle-drawing of $G$ the initial pieces of the edges incident to $v_{0}$ follow each other in the clockwise order $v_{0} v_{1}, \ldots, v_{0} v_{4}$. Then there are two (even) cycles through $v_{0}, v_{1}, v_{3}$ and $v_{0}, v_{2}, v_{4}$ that have no vertex in common other than $v_{0}$. The corresponding two curves cross each other in a small neighborhood of $v_{0}$, contradicting Lemma 2.2.

Suppose next that $G$ contains a subdivision of $K_{3,3}$ with vertex classes $\left\{u_{1}, u_{2}, u_{3}\right\}$ and $\left\{v_{1}, v_{2}, v_{3}\right\}$. Denote this subdivision by $K$. Assume first that the lengths of all nine paths in $K$ connecting the $u_{i}$ 's and the $v_{j}$ 's have the same parity. Deleting from $K$ the point $u_{3}$ together with the three paths connecting it to the $v_{j}$ 's, we obtain a $\Theta$-graph. In view of Lemma 2.3 , it is a converter between $u_{1}$ and $u_{2}$. Similarly, deleting $u_{2}\left(u_{1}\right)$ we obtain a converter between $u_{1}$ and $u_{3}$ ( $u_{2}$ and $u_{3}$, respectively). We say that the type of $u_{i}$ is clockwise or counterclockwise according to the circular order of the initial segments of the paths $u_{i} v_{1}, u_{i} v_{2}, u_{i} v_{3}$ around $u_{i}$. It follows from the definition of a converter that any two $u_{i}$ 's must have opposite types, which is impossible.

There are two other essentially different cases according to the parities of the nine paths forming $K$. It turns out that in both cases one can arrive at a contradiction by showing that there is exactly one pair of points among $u_{1}, u_{2}, u_{3}$ having opposite types.

Proof of Theorem 1.4: In view of the remark at the end of the previous section, the above argument also proves that every bipartite graph that can be drawn as a generalized thrackle is planar. To establish the theorem, we have to show that the reverse of this statement is also true, i.e., every bipartite planar graph $G$ can be drawn as a generalized thrackle. To see this, consider a crossing-free embedding of $G$ in the plane such that

(i) $V(G)=V_{1} \cup V_{2}$, where all points of $V_{1}$ are mapped into the upper half-plane and all points of $V_{2}$ below the line $y=-1$;

(ii) every edge $e \in E(G)$ connects a vertex of $V_{1}$ to a vertex of $V_{2}$, and each piece of $e$ belonging to the strip $-1 \leq y \leq 0$ is a vertical segment.

Now erase the part of the drawing in the strip $-1 \leq y \leq 0$, and replace 
the part in the upper half-plane by its reflection about the $y$-axis. Reconnecting the corresponding pairs of points on the lines $y=-1$ and $y=0$ by straight-line segments, we obtain a drawing of $G$ such that any pair of independent edges meet an odd number of times. This can be turned into a generalized thrackle by slightly modifying the edges in a small neighborhood of their endpoints so as to reverse the circular order of edges around each vertex of $G$.

We could have completed our proof without using Lemma 2.2. The fact that a thrackle contains no subdivion of $K_{5}$ can also be deduced from Lemma 2.3 in a slightly more complicated way.

The proof of Theorem 1.2 also yields the following.

Corollary 3.1 A graph is planar if and only if it has a drawing whose every $\Theta$-subgraph is a converter.

For a related result, see [T70].

\section{Reduction to the bipartite case}

Every graph can be made bipartite by the removal of fewer than half of its edges. It follows from Euler's polyhedral formula that any bipartite planar graph of $n$ vertices has at most $2 n-4$ edges $(n>2)$. If in addition the graph has no cycles of length four then this bound can be replaced by $\lfloor 3 n / 2\rfloor-3$ $(n>3)$. Thus, Theorem 1.4 and Lemma 2.1(i) immediately imply the following.

Corollary 4.1 Let $n>3$. Then

(i) every thrackle of $n$ vertices has at most $3 n-7$ edges;

(ii) every generalized thrackle of $n$ vertices has at most $4 n-9$ edges.

In the rest of this section we sketch how to reduce the bound in Corollary 4.1(i) roughly by $n$.

Let $G$ be a thrackle of $n$ vertices, $n>3$. One can assume that $G$ is not bipartite, otherwise its number of edges cannot exceed $\lfloor 3 n / 2\rfloor-3$. Let $C$ denote a shortest odd cycle of $G$ with length $c$. By Lemma 2.1(i) and by the minimality of $C$, any vertex of $G$ not on $C$ has at most one neighbor belonging to $C$. Hence, there are at most $n$ edges of $G$ which are not on 
$C$ incident to vertices of $C$. It follows from Lemma 2.1(ii) that the graph $G-C$ obtained from $G$ by the removal of all points of $C$ is bipartite. Thus,

$$
|E(G)| \leq|E(G-C)|+n \leq 3(n-c) / 2+n=5 n / 2-3 c / 2 .
$$

One can refine this argument, as follows. The closed curve representing $C$ cuts the plane into a number of cells that can be colored with black and white so that no two cells with a common boundary arc have the same color. Let $b$ and $w$ denote the number of vertices of $G-C$ lying in black and in white cells, respectively. Clearly, $c+b+w=n$, and one can assume without loss of generality that $b \leq w$, so that

$$
b \leq(n-c) / 2 .
$$

Observe that if an edge $e$ connects a point of $C$ to (say) a black vertex, then in a small neighborhood of this point the initial piece of $e$ must be white. There are at most $b$ such edges, and if one removes all of them together with all edges of $C$, the resulting graph (thrackle) becomes bipartite. This with (1) yields the inequality

$$
|E(G)| \leq\lfloor 3 n / 2\rfloor-3+b+c \leq 2 n+c / 2-3 .
$$

Comparing the last two inequalities, we obtain that $|E(G)|<(2+1 / 8) n$.

One can further reduce this bound by utilizing an idea of Conway (see [W69, G93, PRS91, PRS94]). Now we replace each vertex and edge of $C$ by two nearby vertices and edges, respectively. More precisely, we split each vertex $v$ of $C$ into two vertices, $v_{b}$ and $v_{w}$, and connect all black and white neighbors of $v$ not on $C$ to $v_{b}$ and $v_{w}$, respectively. Furthermore, if $v$ and $v^{\prime}$ are two consecutive vertices of $C$, we connect $v_{b}$ to $v_{w}^{\prime}$ and $v_{w}$ to $v_{b}^{\prime}$. It is not hard to see that this construction can be carried out in such a way that the resulting drawing $G^{\prime}$ is a thrackle, which becomes bipartite after the removal of all edges between $v_{b}$ 's and black vertices. Thus,

$$
\left|E\left(G^{\prime}\right)\right|-b=|E(G)|+c-b \leq\lfloor 3(n+c) / 2\rfloor-3,
$$

which implies by (1) that

$$
|E(G)| \leq 2 n-3
$$

as stated in Theorem 1.1. 


\section{Small forbidden configurations}

All of the results in the previous sections were based on parity arguments. Theorem 1.4 shows that if we want to settle Conway's original conjecture, we have to go beyond these methods. In the proof of Theorem 1.1 we were able to explore a property of thrackles that does not hold for generalized thrackles. Namely, we used the fact that a thrackleable graph has no cycle of length four (Lemma 2.1(i)). By excluding some other small configurations that would contradict the thrackle conjecture, one can easily improve the bound in Theorem 1.1. The trouble is that it is quite difficult to find any new non-trivial forbidden subgraph, because even a relatively small graph may have an enormous number of topologically different drawings such that no two edges meet more than once. In this section, we illustrate these difficulties by an example.

Let $\Theta_{3}$ denote a graph consisting of two vertices connected by three internally disjoint paths of length three.

Theorem 5.1 A thrackleable graph cannot contain $\Theta_{3}$ as a subgraph.

For the proof we need some preparation. Let $G$ be a fixed thrackle whose edges are smooth curves. Given two directed edges $e$ and $f$ that do not share an endpoint, we say that $e$ meets $f$ clockwise if at their intersection point a tangent vector to $e$ can be carried into a tangent vector of $f$ by a clockwise turn with angle less than $\pi$.

Let $P=e_{1} e_{2} e_{3} e_{4}$ be a directed path in $G$ with length four, directed towards $e_{4}$. Associate $P$ with a $4 \times 4$ matrix $M$ such that $M_{i j}=0$ if $i=j$ or if $e_{i}$ and $e_{j}$ do not have an interior point in common. Otherwise, let $M_{i j}=1$ or -1 depending on whether $e_{i}$ meets $e_{j}$ clockwise or counterclockwise. Clearly, $M$ is antisymmetric and it is determined by the triple $\left(M_{13}, M_{14}, M_{24}\right)$. This triple is called the type of $P$. It turns out that there are only six possible types:

$a=(1,1,-1) ; b=(1,-1,-1) ; c=(1,-1,1)$;

$A=(-1,-1,1) ; B=(-1,1,1) ; C=(-1,1,-1)$.

Lemma 5.2 Let $e_{1}, e_{2}, \ldots, e_{6}$ be six directed edges of a thrackle that form a simple directed cycle, and let $P_{i}=e_{i} e_{i+1} e_{i+2} e_{i+3}$, where the indices are taken mod 6.

Then type $\left(P_{1}\right)$ type $\left(P_{2}\right) \ldots$ type $\left(P_{6}\right)$ must be one of the following sequences: $A a A a A a, a A a A a A, B b B b B b, b B b B b B$. 
Given a directed path $P=e_{1} e_{2} e_{3} e_{4}$, let the reverse of $P$ be defined as $P^{-1}=e_{4}^{-1} e_{3}^{-1} e_{2}^{-1} e_{1}^{-1}$, where $e_{i}^{-1}$ denotes the same edge as $e_{i}$ but with reversed orientation. If $e_{1} \ldots e_{5}$ is a simple directed path, we say that $P^{\prime}=$ $e_{2} e_{3} e_{4} e_{5}$ can be obtained from $P=e_{1} e_{2} e_{3} e_{4}$ by a shift.

Lemma 5.3 Let $P$ be a path of length four in a thrackle, and assume that $\operatorname{type}(P) \in\{a, b, A, B\}$.

(i) type $\left(P^{-1}\right)=b, a, B$ or $A$ according to whether type $(P)=a, b, A$ or $B$.

(ii) If $P^{\prime}$ can be obtained from $P$ by a shift and type $\left(P^{\prime}\right) \in\{a, b, A, B\}$, then type $(P)$ type $\left(P^{\prime}\right)$ must be one of the following six pairs: $a A, a B, b B, A a, A b, B b$.

Proof of Theorem 5.1: Assume that there is a thrackle containing $\Theta_{3}$ as a subgraph. By Lemma 5.2, the type of every directed path of $\Theta_{3}$ belongs to the set $\{a, b, A, B\}$. Consider a path $P$ whose type belongs to $\{b, B\}$. (If $P$ does not satisfy this condition then its reverse does.) Observe that the topology of $\Theta_{3}$ allows us to transform $P$ into its reverse by a series of shifts. It follows from Lemma 5.3(ii) that the types of all paths obtained during this process belong to $\{b, B\}$. However, by Lemma 5.3(i), type $\left(P^{-1}\right) \in\{a, A\}$, which is a contradiction.

\section{References}

[BF88] L. Babai and P. Frankl, Linear Algebra Methods in Combinatorics, Part I, Tech. Report, University of Chicago.

[B69] E.R. Berlekamp, On subsets with intersections of even cardinality, Canadian Math. Bull. 12, 363-366.

[BE48] N.G. de Bruijn and P. Erdős, On a combinatorial problem, Proc. Konink. Nederl. Akad. Wetensch., Ser. A 51, 1277-1279 (=Indagationes Math. 10, 421-423.)

[DETT94] G. Di Battista, P. Eades, R. Tamassia, and I.G. Tollis, Algorithms for drawing graphs: an annotated bibliography, Computational Geometry: Theory \& Applications 4, 235-282.

[FS35] W. Fenchel and J. Sutherland, Lösung der Aufgabe 167, Jahresbericht der Deutschen Mathematiker-Vereinigung 45, 33-35.

[F40] R. A. Fisher, An examination of the different possible solutions of a problem in incomplete blocks, Annals of Eugenics (London) 10, $52-75$. 
[GJ83] M.R. Garey and D.S. Johnson, Crossing number is NP-complete, SIAM J. Algebraic Discrete Methods 4, 312-316.

[G75] R.L. Graham, The largest small hexagon, J. Combinatorial Theory 18, 165-170.

[G93] J. Green-Cottingham, Thrackles, Surfaces and Drawings of Graphs, Doctoral Dissertation, Clemson University.

[G72] R. K. Guy, Crossing numbers of graphs, in: Graph Theory and Applications, Lecture Notes in Mathematics, Vol. 303, Springer-Verlag, $111-124$

[HP34] H. Hopf and E. Pannwitz, Aufgabe Nr. 167, Jahresbericht der Deutschen Mathematiker-Vereinigung 43, 114.

[KST54] T. Kővári, V. T. Sós, and P. Turán, On a problem of K. Zarankiewicz, Colloqium Mathematicum 3, 50-57.

[LST94] J. A. de Loera, B. Sturmfels, and R. R. Thomas, Gröbner bases and triangulations of the second hypersimplex, Combinatorica, to appear.

[PA95] J. Pach and P.K. Agarwal, Combinatorial Geometry, J. Wiley, New York.

[PRS91] B. Piazza, R. Ringeisen, and S. Stueckle, Properties of nonminimum crossings for some classes of graphs, in: Graph Theory, Combinatorics, and Applications, Vol. 2 (Y. Alavi et al., eds.), J. Wiley, New York, 975-989.

[PRS94] B. Piazza, R. Ringeisen, and S. Stueckle, On Conway's reduction of the thrackle conjecture and some associated drawings,, to appear.

[RSP91] R. Ringeisen, S. Stueckle, and B. Piazza, Subgraphs and bounds on maximum crossings, Bulletin ICA 2, 33-46.

[T77] P. Turán, A note of welcome, J. Graph Theory 1, 7-9.

[T70] W.T. Tutte, Toward a theory of crossing number, J. Combinatorial Theory 8, 45-53.

[WB78] A.T. White and L.W. Beineke, Topological graph theory, in: Selected Topics in Graph Theory (L. Beineke, R. Wilson, eds.), Academic Press, London, 15-49. 
[W69] D. R. Woodall, Thrackles and deadlock, in: Combinatorial Mathematics and Its Applications (D.J.A. Welsh, ed.), Academic Press, London, 335-348.

[W72] D. R. Woodall, Open problems, in: Combinatorics, Proc. Conference on Combinatorial Mathematics (D.J.A. Welsh, D.R. Woodall, eds.), Institute of Mathematics and Its Applications, Southend-on-Sea, Essex, England, 341-350. 